University of Wollongong

Research Online

Australian Institute for Innovative Materials -

Papers

Australian Institute for Innovative Materials

$1-1-2016$

Smart fabrics and networked clothing: recent developments in CNT-based fibers and their continual refinement

Javad Foroughi

University of Wollongong, foroughi@uow.edu.au

Teodor E. Mitew

University of Wollongong, tmitew@uow.edu.au

Philip O. Ogunbona

University of Wollongong, philipo@uow.edu.au

Raad Raad

University of Wollongong, raad@uow.edu.au

Farzad Safaei

University of Wollongong, farzad@uow.edu.au

Follow this and additional works at: https://ro.uow.edu.au/aiimpapers

Part of the Engineering Commons, and the Physical Sciences and Mathematics Commons

Research Online is the open access institutional repository for the University of Wollongong. For further information contact the UOW Library: research-pubs@uow.edu.au 


\title{
Smart fabrics and networked clothing: recent developments in CNT-based fibers and their continual refinement
}

\author{
Abstract \\ Virtually all human cultures routinely wear garments or some sort of attire, both for protection against the \\ elements and as part of a complex social display of everything from class status to gender to the act of \\ belonging to a particular subculture. Smart garments, in addition to performing their social functions, can \\ act as transmitters, sensors, or energy-harvesting entities. Furthermore, such garments may also possess \\ some processing capability, enabling them to perform a variety of tasks, perhaps in conjunction with \\ cloud-based server resources. \\ Disciplines \\ Engineering | Physical Sciences and Mathematics

\section{Publication Details} \\ Foroughi, J., Mitew, T. E., Ogunbona, P. O., Raad, R. \& Safaei, F. (2016). Smart fabrics and networked \\ clothing: recent developments in CNT-based fibers and their continual refinement.. IEEE Consumer \\ Electronics Magazine, 5 (4), 105-111.
}




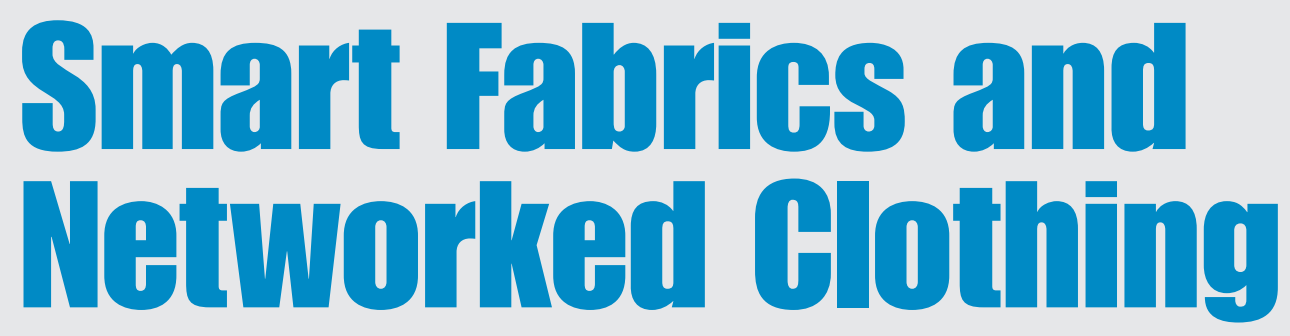

By Javad Foroughi, Teodor Mitew, Philip Ogunbona, Raad Raad, and Farzad Safaei

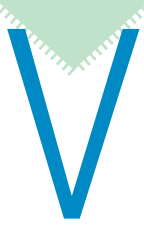

IRTUALLY ALL HUMAN CULTURES ROUTINELY WEAR GARMENTS OR SOME SORT of attire, both for protection against the elements and as part of a complex social display of everything from class status to gender to the act of belonging to a particular subculture. Smart garments, in addition to performing their social functions, can act as transmitters, sensors, or energy-harvesting entities. Furthermore, such garments may also possess some processing capability, enabling them to perform a variety of tasks, perhaps in conjunction with cloud-based server resources.

The applications for such fabrics are many, ranging from uses in specific industries to the almost infinite mundane scenarios of daily life. One potential application is real-time tracking of the location and vital signs of the wearer, which may be particularly relevant for first responders (such as police and firefighters), the military, miners, and industrial workers. In most cases, tracking a garment is preferred, because carried devices, in addition to being inconvenient, can be lost, dropped, or malfunction during emergencies. Collected data are transmitted wirelessly to a remote server where they can be used, in conjunction with information from other

Digital Object Identifier 10.1109/MCE.2016.2590220 Date of publication: 22 September 2016 
people, to gain insight into key health issues or hazards faced by the wearer. The contemporary precursors of networked clothing are today's wristbands, which mainly focus on fitness and health monitoring. There are also several companies that have ventured into smart clothing that target serious athletes.

The obvious flip side of using smart fabrics is the potential misuse of their capabilities. For example, networked clothes are perennially at risk of being accessed, defaced, or otherwise exploited by hackers. There is also the permanent risk of unwanted or unintended body-surveillance and the erosion of any remaining sense of privacy. The ability to, as it were, switch off the fabric by the end user is essential, but may not be sufficient, since the entire garment may act as an antenna. The social implications of a pervasive Internet in which humans are mediated through clothing promise to redefine fundamental assumptions about social relationships and raise new questions about privacy, ownership of data, and the tradeoff between utility and security.

One of the challenges for the manufacture of smart fabrics is achieving a seamless and invisible integration of the required electronics into the fabric. Consequently, materials researchers need to produce fibers with the desired electronic functionality without compromising strength, comfort, and aesthetic appeal. At the same time, the development of these electronic textiles forces a rethinking of the design choices adopted in implementing the electronic circuits and communication systems for optimum performance. In particular, the antenna system required for the transmission of data deviates from convention. There are also implications for the sensing, computational, and storage units.

Recent developments in advanced materials and wireless communications show significant promise in achieving the above goals. In particular, researchers have shown that carbon nanotubes (CNTs), which are seamless, cylindrical, hollow fibers of a single sheet of pure graphite, can be fabricated into many useful forms. In the context of this article, useful forms, such as sheets or yarns, can be fabricated and embedded in garments, fabrics, and other everyday objects [1]-[4]. These materials, either in pristine form or in combination with other polymers, metals, or graphene, exhibit superior mechanical and electronic properties [1], [5], [6]. Most importantly, experimental assessment of their high-frequency properties in the gigahertz range [7] shows their potential for incorporating radiating elements and antennas in garments.

The aim of this article is to provide an overview of the exciting new research in the area of smart fabrics, with particular focus on recent developments in CNT-based fibers and their continual refinement to make them suitable as embedded antennas or actuators.

$<\mathrm{AU}$ : Please note that the text outlining the article was removed as per magazine style.>

\section{POTENTIAL APPLICATIONS}

Ubiquitous wireless infrastructure and devices have literally transformed our society in recent decades. Highly conductive
CNT materials that can be embedded in everyday objects and are capable of operating at high frequencies may be suitable for a range of communications and electronic applications. New CNT-based materials can be used to create invisible antenna patches incorporated in garments or in the plastic casing of devices. For example, a typical patch of 20 antenna elements at $60 \mathrm{GHz}$ will be around $50 \mathrm{~mm} \times 5 \mathrm{~mm}$ and could be used to facilitate low-power and high-speed communication at home, the office, or a work site.

Importantly, this ambient wireless infrastructure supports communication in and around the body (so-called body area networking) for medical and health applications. The antenna array incorporated in garments will create the communication link between sensors and devices in and on the body (e.g., drug-delivery microdevices injected into the bloodstream or sensors incorporated in e-skin) and will connect the individual to the household communication hub (Figure 1). This connectivity is low power, high speed, and always on, enabling a range of applications not only for health and well-being but for entertainment, interaction, and control. A challenging application domain for smart garments, with significant potential benefits, is for monitoring, tracking, and communication with personnel working in mines or other hazardous and hard-to-reach spaces. In this scenario, seamless integration of functionality into work garments and nondependence on a power source (so it is always active and attached to the personnel) will result in meeting the stringent requirements for the design of materials and circuitry.

\section{CNT FIBERS AND FABRICS}

CNTs already show promising performance for myriad applications, including supercapacitors, actuators, and lightweight electromagnetic shields (Figure 2). Since their first observation nearly three decades ago by Iijima [8], CNTs have been the focus of considerable research. Numerous investigators have since reported remarkable physical and mechanical properties for this new form of carbon. From unique electronic

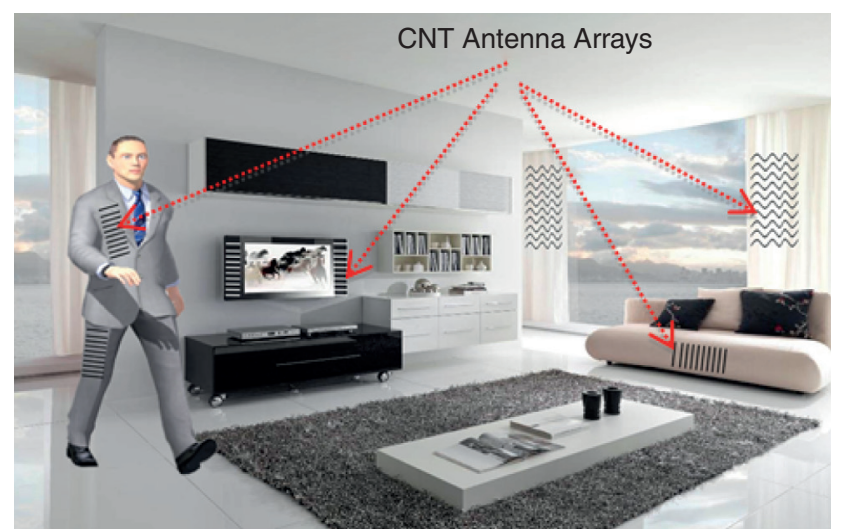

FIGURE 1. An artist's conception of CNT-embedded massive antenna arrays in a home networking scenario. For clarity, the antenna arrays are shown large and visible, while in practice the antenna patches would be small and invisible. $\angle A U$ : From where was this image obtained, and do you have permission from the source to use it?> 


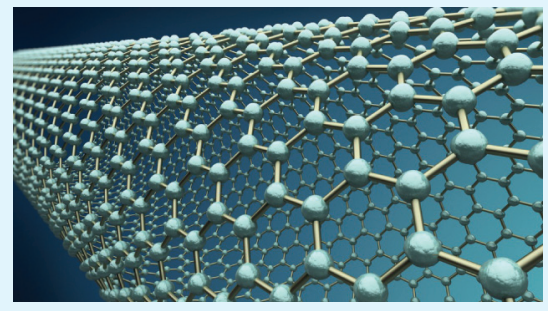

(a)

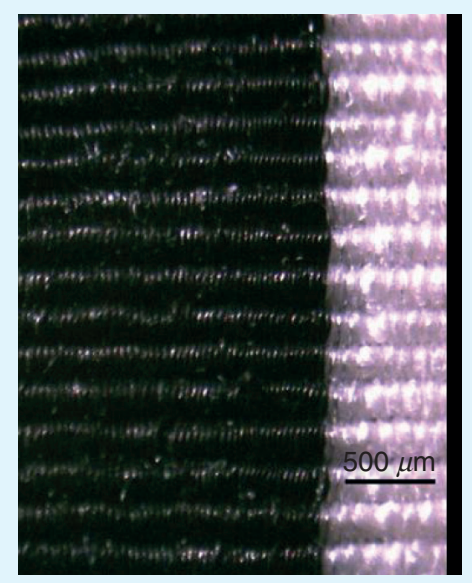

(c)

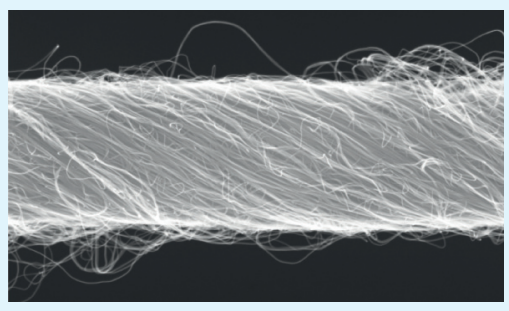

(b)
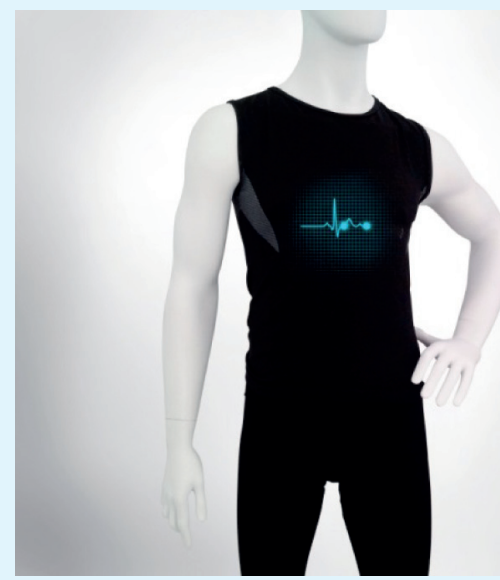

(d)

FIGURE 2. A schematic illustration of fabricating smart textiles: (a) a carbon nanotube, (b) twisted yarn, (c) the woven textile, and (d) a smart garment. <AU: From where were these images obtained, and do you have permission from the source to use them?>

properties and a thermal conductivity higher than diamond to mechanical properties where their stiffness, strength, and resilience exceed any current material, CNTs offer tremendous opportunities for the development of fundamentally new material systems. CNTs have attracted enormous attention for their use in a wide variety of applications, such as in nanoelectronic devices, probe tips for scanning probe microscopes, actuators, and sensors and in the automotive and aerospace industries for the dissipation of electrostatic charges [9]-[11]. Single-walled CNTs and multiwalled CNTs are effectively rolled-up graphene sheets and share the exceptional mechanical properties of graphene. Theoretical and experimental results have shown that this carbon form has an extremely high elastic modulus, greater than $1 \mathrm{TPa}$ (the elastic modulus of diamond is $1.2 \mathrm{TPa}$ ); a thermal conductivity about twice as high as diamond; an electric current-carrying capacity 1,000times higher than copper wire; and a reported strength ten to 100-times higher than the strongest steel at a fraction of the weight for single-walled CNTs. Owing to CNTs' structural characteristics and their electrical and mechanical properties, one of the most important opportunities in the future is the emergence of a new generation of bicomponent materials for various applications. CNTs may result in a whole new class of advanced materials.

CNTs can be grown as a forest using a variety of methods, from which they can be drawn off and twisted into a CNT yarn (solid-state) for the fabrication of three-dimensional (3-D) structures through braiding, weaving, sewing, and knitting [4], [12]-[14]. In particular, researchers have shown that doped yarns comprising single- and fewwalled nanotubes can provide a higher gravimetric dc electrical conductivity than copper wire [15]. However, the mechanical properties of as-prepared fibers are not suitable for real application in woven or knitted textiles. Hence, fabrication of CNTs into the textile, achieving traditional textile properties, such as considerable softness, strength, flexibility, texture, and washability, is still a challenge.

\section{CNT AND METALLIC ANTENNAS FOR SMART FABRICS}

Wearable and on-body wireless devices are not new. Bluetooth can be considered one of the first standards to have a major part of its appeal in the wearable sphere. Since then, a push has been made to integrate transmitters and receivers into garments and other wearable devices, such as helmets and uniforms, and some experiments have integrated radio frequency identification (RFID) tags into the body itself in the form of under-theskin implants. Current efforts are focused on the high-frequency range (e.g., the gigahertz range) to ensure that antennas are small and that their integration into, say, clothing tags or buttons, is possible. At lower frequencies (e.g., 20 $\mathrm{MHz}$ ), the size of the antenna patches must be larger. However, these frequencies may be more suitable for location tracking, as the signal can penetrate rocks and other high-density structures. It is also possible to energize the garment through a wireless signal with higher-powered transmitters at distances of 20-30 m and potentially even longer.

Metals have been the traditional materials used for antennas because of their low impedance and, hence, low loss, resulting in their being efficient radiators of energy. But metals are hard to integrate into garments due to their corrosion and inflexibility above certain sizes. In 2005, Hansen observed that CNT materials could be "metallic or semiconducting, depending on geometry" [16]. However, the theoretical work by Hansen and Attiya showed that transmission frequencies should be on the order of hundreds of gigahertz and the antenna length on the order of tens of micrometers [17]. Such high-gigahertz-frequency bands have only recently been proposed for $5 \mathrm{G}$ communications $(7-110 \mathrm{GHz})$. As a result, this has limited the adoption of these CNT materials and provided an indication that CNT communications might be limited to short-range applications. <AU: Please check 
whether the preceding edited sentence conveys the intended meaning.>

The application of CNTs at very high optical frequencies has recently been reported in Nature Nanotechnology as the first operating CNT optical rectenna [18]. A rectenna is an antenna that operates in the optical frequencies. The authors used a CNT sheet as the receiving antenna connected directly to a photodiode (switching at a speed of $1 \mathrm{PHz}$ ) to drive a current through it. Such a device will allow communications at very high bit rates.

Dipoles or straight-wire antennas are not the only form, and it is shown that by using a patch configuration, more reasonable operating frequencies may be possible. A patch antenna is just a flat piece of material that allows energy to radiate out from its surface. Recent patch antennas made from CNTs show promise at operating in the 4-9-GHz range with good gain [19], [20].

With respect to applicability at lower frequencies (below $4 \mathrm{GHz}$ ), Nepa and Rogier recently published an extensive survey focused on frequencies ranging from $70 \mathrm{MHz}$ to a few hundreds of megahertz [21]. These frequencies, while not affording high-bandwidth communication, tend to be more suitable for longer range communication and communication through walls and other materials. However, many of the antennas noted in the survey rely simply on copper tape or different types of metallic material that is either woven into or embroidered on the inside of clothing. These antennas can measure more than $1 \mathrm{~m}$ in length or cover areas up to $60 \mathrm{~cm} \times 60 \mathrm{~cm}$. Therefore, development of CNT-based antennas for low-frequency applications is still an open research question.

Multiantenna systems have also been implemented that allow operating frequencies between $2 \mathrm{MHz}$ and $2 \mathrm{GHz}$. They come in a variety of forms, mostly aimed at military and first-responder applications. In these applications, effectively all the clothing, from the helmet down to the boots, is covered by antenna elements, allowing the responder to communicate at very low to reasonably high frequencies. That being said, the current solutions are based on the flexible application of metal-based yarns.

\section{CHALLENGES IN DEVELOPING CNT-EMBEDDED ANTENNAS AND DEVICES}

The development of suitable forms and compositions of CNT-based materials for inclusion in smart textiles as antenna arrays and/or electronic textiles seems plausible but requires the research community to tackle a number of significant challenges.

\section{TUNING THE ELECTROMAGNETIC RADIATION PROPERTIES OF CNT FIBERS}

As mentioned in the previous section, with respect to electromagnetic radiation properties, the current CNT fibers are not as suitable as their metallic counterparts, especially at lower frequencies. However, an individual CNT has low scattering, high current-carrying capacity, and resistance to electromigra- tion [22], [23]. More research, therefore, is needed to improve the fabrication process of yarns to take advantage of these characteristics. In particular, there is a need for better modeling and characterization of electron mobility properties; resistive, capacitive, and inductive characteristics; and nonlinear effects. A potentially promising approach to improve their performance is by the incorporation of graphene (or other suitable conductors) within the CNT yarn and by creating new 3-D forms by, for example, weaving, braiding, or knitting.

\section{ANTENNA SELECTION AND SWITCHING}

Using a massive antenna array can improve the wireless communications performance and power consumption through at least two mechanisms.

1) Multiple antennas can be used to focus the radiation toward a specific device or region (referred to as beamforming). Consequently, higher transmission bit rates can be achieved because the transmission power is not wasted in radiating the signal in all directions.

2) If the separation distance between the antennas is sufficient (with respect to the size of the wavelength), then the wireless communication channels between each antenna and the receiver become more or less independent. This property can be utilized by the transmitter to improve the data delivery. For example, by suitably processing the multiple versions of the signal received from different paths, the chances of error-free decoding is improved. Alternatively, the transmitter may sense each of the available channels and transmit using a particular subset of antennas that have a better channel to the receiver, with less interference and noise. (This is referred to as antenna switching) [24].

Both techniques may be used by smart fabrics, but the latter approach, in particular, looks promising in view of the recent development of twist-spun CNT yarns that can be used as a muscle to provide fast, high-force, high-strain torsional and tensile actuation that is reversible for over a million cycles [17], [36], [37]. Using similar concepts, it might be possible to develop antenna switching and adapt the radiation pattern of the antennas to wireless channel variations.

\section{POWER SUPPLY AND POWER SCAVENGING}

Batteries are the only current viable power source, but their weight and bulk affect wearer comfort and garment aesthetics. In addition, batteries have limited energy capacity and need replacing or recharging regularly. Therefore, a growing research effort aims to harness electrical energy directly from the wearer, using body movement or heat to self-power the devices. While this research area is still in its infancy, preliminary studies have already identified potentially useful flexible piezoelectric materials that convert movement to usable electrical power that may be coupled with supercapacitors and batteries to store the harvested energy [25]. Self-powered wireless sensors, typically using thermal energy harvesting, have already been successfully deployed for monitoring temperature, pressure, and chemical species in 
industry. The challenge now is to develop such systems for garments.

Typical power requirements of sensors are in the $20-\mathrm{mW}$ range, while low-energy Bluetooth transmitters require up to $10-500 \mathrm{~mW}$ for operation. For example, a commercially available stretchable strain/pressure sensor based on elastomer membranes and with Bluetooth requires an input power of $200 \mathrm{~mW}$. Recent studies have shown that such power requirements can be achieved with flexible, lightweight transducers. Dielectric elastomer stacks incorporated into the heel of a shoe generate $\sim 1 \mathrm{~J}$ per step, and small $\left(<0.5 \mathrm{~cm}^{3}\right)$, ceramic-based piezoelectric units have been shown to generate $8 \mu \mathrm{W}$ from heartbeat vibrations and $1.6 \mathrm{~mW}$ from lowfrequency bending. Using hybrid piezoelectric polymer materials with a reported power output of $78 \mathrm{~mW} / \mathrm{cm}^{3}$, we will need $\sim 80 \mathrm{~m}$ of these fibers to generate $200 \mathrm{~mW}$ [26]. This area could be easily accommodated as a $2-3-\mathrm{cm}$-wide band around the chest so as to couple with breathing movements. It is likely that the power generated would be used to charge a small battery, with power used intermittently for sensing and data transmission. This concept has been successfully demonstrated using nonflexible piezoelectric patches on T-shirts that harness sound waves at rock concerts [27].

To produce high-performance stretchable piezoelectric fibers, a number of fundamental challenges must be addressed. First, all piezoelectrics are rigid materials, and strategies need to be employed to access high-tensile strains by forming corrugated, coiled, or highly porous structures with compatible electrodes and deformation modes that produce a net charge separation. Another challenge is to ensure that the electrode materials remain undamaged and in close contact with the active material during its deformation. Finally, inevitable imperfections associated with electrode contact points result in spurious and parasitic impedance variations across the fabric that may reduce the efficiency of power harvesting. There may also be significant variations over time in the electrical characteristics of these contact points due to movements and deformations. <AU: Please check whether the preceding edited sentence conveys the intended meaning.> The topology of the interconnections must also be carefully designed to ensure proper alignment of the phases of the myriad power sources both spatially and temporally.

With respect to power requirements, a challenging scenario will be to support a satellite transceiver (satellite phone). This functionality will be of particular importance for tracking, rescue, and military operations. Current satellite phones, using low earth orbit satellites, require $500-1,000 \mathrm{~mW}$ of power to access the service from anywhere in the world. An ultimate goal will be to provide continuous connectivity using only the power harnessed from the piezo-fabrics.

\section{ANALYTICS OF SMART NETWORKED CLOTHING}

The availability of massive amounts of machine-generated data has brought on the era of so-called big data. The scenario that will be created by smart and networked clothing is more akin to what is called small data, wherein scant, near- instantaneous data are acquired and used to provide advice or make a decision. The challenge is how to adapt models learned from different environments in conjunction with current data to provide the required actionable advice.

Arguably, a lot of the "smarts" of smart clothing will derive from the ability to acquire data and then process and provide contextual decisions. This is in conjunction with the ability to perform duplex communication to facilitate the decision-making process. In the case of smart clothing, the state of the body is being acquired and presented and contextual decisions are being made. In a conventional machinelearning environment, there is a vast amount of data from which to learn, and there is the inherent assumption that the underlying statistical distributions of the training and test data are identical. There are also other assumptions about the labels (identifiers) associated with the individual data elements in the training and test data. The assumptions of equality of distributions and labels are often not valid in real-life situations. The implications of these variations are especially important when the eventual decision is directed at humans. The challenge is how to adapt a learned model based on some previously collected data and in a different scenario to the statistics of current data from a new person. This is usually accomplished in a transfer learning paradigm - a current hot topic in machine learning. The question then arises as to how to use generic data to provide personalized decisions.

\section{LIVING WITH NETWORKED CLOTHES}

The continuous monitoring entailing the notion of smart fabrics has profound implications not only for humans but for nonhumans, everyday objects, and material settings. At a very basic level, these implications have to do with the already emergent modes of pervasive surveillance, as they have the obvious potential to directly affect and modulate human behavioral patterns. Wearable, always on, always connected fabrics quite clearly lead to clothes residing permanently in the Internet of Things (IoT) and to humans operating within a mode of constant biosurveillance. For example, imagine a scenario in which office workers are continuously monitored for pulse, body temperature, and movement, while the data are aggregated and contextually analyzed for patterns. However, while it could be argued that surveillance concerns are primarily epistemological, in that they involve issues of control over access to monitoring information [28], the stakes change radically when we take into account the concurrent emergence of an animate IoT environment populated by sociable objects that are no longer passive and inert [29]. Arguably, the implications facing the emergence of smart, permanently networked clothes and fabrics are not confined only to epistemological concerns but amount to a paradigm shift into a whole new set of ontological issues at the level of the body itself [30].

How did we come to this point? Back in the early days of the Internet, Mark Weiser envisaged pervasive, ubiquitous computing as an environment where the technical apparatus recedes into an invisible, always unnoticeable material back- 
ground of daily life [31]. <AU: Please check whether the preceding edited sentence conveys the intended meaning.> Today, the communication technologies building the IoT consist of stacked layers of fiber and wireless networks; wireless frequency standards for mesh and near-field networking such as ZigBee, Bluetooth, RFID, and near-field communication (NFC); and a rapidly growing number of human-oriented devices able to communicate across one or several of these layers using proprietary or open-source software. Furthermore, underneath this IoT layer, interfacing with and visible to humans, there lies a vast realm of machine-to-machine (M2M) communication standards, technologies, and software, dwarfing the human-oriented IoT layer in size and complexity [32]. While currently the most prevalent human IoT interfaces are smartphone devices capable of data exchange across 3G/4G, Wi-Fi, Bluetooth, and NFC, the field is rapidly expanding into wearables, such as Fitbit and other networked objects to be worn on one's body or interfaced with in some other way [33].

It is worthwhile to briefly consider the logistics of connecting a random object or piece of clothing to the Internet. To begin with, IoT connectivity implies that clothes acquire a network address, thus becoming uniquely identifiable, and broadcast sensory data either remotely to the cloud or in an M2M mesh of devices. The sensory layer, if there is one attached, allows clothes to dynamically register changes to their environment and transmit that information over the network. Finally, an IoT-enabled piece of clothing has the ability to process that information locally or in the cloud, and act upon it independently from human input [34]. From a data perspective, every garment equipped in this way becomes context-aware, where context is understood as the triad of location-identity-state [35]. As a result, such clothes have a unique identity and are capable of dynamically engaging with and registering changes to their location and state.

Now, consider already existing and overtly sociable objects such as the Amazon Echo. Here, the IoT manifests itself through physical objects acting as components of a system of pervasive monitoring and control, augmenting materiality with a layer of Internet connectivity. Arguably, from a human perspective, the tangible result is a seemingly animate materiality, populated by objects often behaving as social heteroclites in that they transgress the social boundaries set for them [29].

In addition, the contextual data aggregated by a networked garment would most probably be transmitted to and indexed in a remote database, from the perspective of which the contextual data themselves are the object. This is the case because, from the point of view of that remote database, the garment exists as an aggregate of location-identity-state updates. Suddenly, the piece of clothing has morphed into an information space. The logical endpoint of this dynamic is that any IoT entity-be it a smart garment or a human being-is a set of database relationships first and foremost, and a material entity now and then [36], [37]. It only follows that, if from a database perspective humans and smart fabrics are primarily information spaces, their ontological status shifts dramatically, to the extent that, as Rob van Kranenburg quips "you will have more in common with your car than with your neighbor" [38].

The emergence of smart garments, and therefore inevitably that of networked clothes, promises to even further problematize this state of affairs. When wearables first appeared on the market in scale, they quickly led to unpredictable sociocultural developments such as the quantified-self movement, the move to biosurveillance, and the fetishization of body data as a new aesthetic form of self-expression [33]. Living in an environment pervasively populated by networked clothes, fabrics, textiles, and other materials is fraught with the promise of a new aesthetics of the body as a fully networked object. As wearables have demonstrated, such an environment will be quickly normalized and woven into the existing texture of our sociocultural practices, ranging from daily routines and fashion choices, to the extreme and risky realities of the military, heavy industry, and emergency response teams.

\section{ABOUT THE AUTHORS}

Javad Foroughi <AU: Kindly provide e-mail in parentheses and then brief bio information.>

Teodor Mitew (tmitew@uow.edu.au) <AU: Kindly provide brief bio information.>

Philip Ogunbona <AU: Kindly provide e-mail in parentheses and then brief bio information.>

Raad Raad <AU: Kindly provide e-mail in parentheses and then brief bio information.>

Farzad Safaei <AU: Kindly provide e-mail in parentheses and then brief bio information.>

\section{REFERENCES}

[1] J. Foroughi, G. M. Spinks, S. R. Ghorbani, M. E. Kozlov, F. Safaei, G. Peleckis, G. G. Wallace, and R. H. Baughman, "Preparation and characterization of hybrid conducting polymer-carbon nanotube yarn," Nanoscale, vol. 4, no. 3, pp. 940-945, 2012.

[2] J. Foroughi, et al. "Torsional carbon nanotube artificial muscles," Science, vol. 334, no. 6055, pp. 494-497, 2011.<AW - Kindly replace "et al." with the rest of the article author names.>

[3] M. D. Lima, et al. "Electrically, chemically, and photonically powered torsional and tensile actuation of hybrid carbon nanotube yarn muscles," Science, vol. 338, no. 6109, pp. 928-932, 2012. <A山; Kindly replace "et al." with the rest of the article author names

[4] M. Zhang, et al. "Strong, transparent, multifunctional, Carvon nanotube sheets," Science, vol. 309, no. 5738, pp. 1215-1219, 2005. <AU: Kindly replace "et al." with the rest of the article author names.>

[5] J. Foroughi, et al. "Highly conductive carbon nanotube-graphene hybrid yarn," Advanced Functional Materials, vol. 24, no. 37, pp. 58595865, 2014. <AU: Kindly replace "et al." with the rest of article author names.>

[6] M. F. De Volder, et al. "Carbon nanotubes: Present and future commercial applications," Science, vol. 339, no. 6119, pp. 535-539, 2013. <AU: Kindly replace "et al." with the rest of the article author names.>

[7] S. M. Abbas, et al. "Microwave characterization of carbon nanotube yarns for UWB medical wireless body area networks," IEEE Trans. Microwave Theory Techniques, vol. 61, no. 10, pp. 3625-3631, 2013. Kindly replace "et al." with the rest of the article author names.> [8] S. Iijima, "Helical microtubules of graphitic carbon," Nature, vol. 354 , no. 6348 , pp. 56-56, 1991. 
[9] R. H. Baughman, et al. "Carbon nanotube actuators," Science, vol. 284, no. 5418, pp. 1340-1344, 1999. <AU: Kindly replace "et al.” with the rest of the article author names.>

[10] N. Ferrer-Anglada, M. Kaempgen, and S. Roth, "Transparent and flexible carbon nanotube/polypyrrole and carbon nanotube/polyaniline $\mathrm{pH}$ sensors," Physica Status Solidi (B), vol. 243, no. 13, pp. 3519-3523, 2006.

[11] L. Bokobza, "Multiwall carbon nanotube elastomeric composites: A review," Polymer, vol. 48, no. 17, pp. 4907-4920, 2007.

[12] R. H. Baughman, A. A. Zakhidov, and W. A. de Heer, "Carbon nanotubes: The route toward applications," Science, vol. 297, no. 5582 , pp. 787-792, 2002.

[13] S. L. Edwards, et al. "Tubular micro-scale multiwalled carbon nanotube-based scaffolds for tissue engineering," Biomaterials, vol. 30, no. 9, pp. 1725-1731, 2009. <AU: Kindly replace "et al." with the rest of the article author names.>

[14] M. Zhang, K. R. Atkinson, and R. H. Baughman, "Multifunctional carbon nanotube yarns by downsizing an ancient technology," Science, vol. 306, no. 5700, pp. 1358-1361, 2004.

[15] N. Behabtu, et al. "Strong, light, multifunctional fibers of carbon nanotubes with ultrahigh conductivity," Science, vol. 339, no. 6116, pp. 182-186, 2013.

[16] G. W. Hanson, "Fundamental transmitting properties of carbon nanotube antennas," IEEE Trans. Antennas Propagat., vol. 53, no. 11, pp. 3426-3435, 2005.

[17] A. M. Attiya, "Lower frequency limit of carbon nanotube antenna," Progress In Electromagn. Res., vol. 94, pp. 419-433, 2009.<AU: Kindly provide issue number and/or month of publication.>

[18] A. Sharma, et al. "A carbon nanotube optical rectenna," Nature Nanotechnol., vol. 10, no. 12, pp. 1027-1032, 2015. <AU: Kindly replace "et al." with the rest of the article author names.>

[19] H. Zahir, et al. "Design fabrication and characterisation of polyaniline and multiwall carbon nanotubes composites-based patch antenna," IET Microwaves, Antennas Propagat., vol. 10, no. 1, pp. 88-93, 2016.<AU: Kindly replace "et al." with the rest of the article author names.> [20] S. D. Keller and A. I. Zaghloul, "Radio frequency anisotropic patch antenna and polarization selective surface," U.S. Patent 20160126633 2016.

[21] P. Nepa and H. Rogier, "Wearable antennas for off-body radio links at VHF and UHF bands: Challenges, the state of the art, and future trends below 1 GHz," IEEE Antennas Propagat. Mag., vol. 57, no. 5, pp. 30-52, 2015.

[22] P. Jarosz, et al. "Carbon nanotube wires and cables: Near-term applications and future perspectives," Nanoscale, vol. 3, no. 11, pp. 4542-4553, 2011. <AU: Kindly replace "et al." with the rest of the article author names.>

[23] A. Lekawa-Raus, et al. "Electrical properties of carbon nanotube based fibers and their future use in electrical wiring," Advanced Func- tional Materials, vol. 24, no. 24, pp. 3661-3682, 2014. <AU: Kindly replace "et al." with the rest of the article author names.>

[24] N. P. Le and F. Safaei, "Antenna selection strategies for MIMOOFDM wireless systems: An energy efficiency perspective," IEEE Trans. Veh. Technol., vol. 65, no. 4, pp. 2048-2062, 2016.

[25] M. Lee, et al. "A hybrid piezoelectric structure for wearable nanogenerators," Advanced Materials, vol. 24, no. 13, pp. 1759-1764, 2012.<AU: Kindly replace "et al." with the rest of the article author names.>

[26] L. Gu, et al. "Flexible fiber nanogenerator with $209 \mathrm{~V}$ output voltage directly powers a light-emitting diode," Nano Lett., vol. 13, no. 1, pp. 91-94, 2012. <AU: Kindly replace "et al." with the rest of the article author names.>

[27] P. Ridden. (2011, June 22). Sound Charge t-shirt tops up mobile devices using sound [Online]. Available: http://www.gizmag.com/orangeand-gotwind-announce-sound-charge-device-charging-tshirt/18994/

[28] N. K. Hayles, "RFID: Human agency and meaning in informationintensive environments," Theory, Culture Soc., vol. 26, no. 2-3, pp. 47-72, 2009.

[29] T. Mitew, "Do objects dream of an Internet of Things?" Fibreculture J., vol. 2014, no. 23, pp. 1-25, 2014

[30] I.Bogost, Alien Phenomenology, or What It's Like to Be a Thing. Minneapolis, MN: University of Minnesota Press, 2012.

[31] M. Weiser and J. S. Brown, "The coming age of calm technology," in Beyond Calculation: The Next Fifty Years of Computing, P. J. Denning and R. M. Metcalfe, Eds. Göttingen, Germany: Copernicus, 1997, pp. $75-85$.

[32] A. Aijaz and A. H. Aghvami, "Cognitive machine-to-machine communications for Internet-of-Things: A protocol stack perspective," IEEE Internet of Things J., vol. 2, no. 3, pp. 103-112, 2015.

[33] D. Lupton, "Understanding the human machine," IEEE Technol. Soc. Mag., vol. 32, no. 4, pp. 25-30, 2013.

[34] Yan, L., et al. Eds., The Internet of Things: From RFID to the NextGeneration Pervasive Networked Systems. New York: Auerbach, 2008. <AU: Kindly replace "et al." with the rest of the editor names.>

[35] G. D. Abowd, et al. "Towards a better understanding of context and context-awareness," in Handheld and Ubiquitous Computing: Proceedings of the First International Symposium, HUC "99. Berlin: Springer, 1999, pp. 304-307. <AU: Kindly replace "et al." with the rest of the article author names.>

[36] B. Sterling, The Epic Struggle of the Internet of Things. Moscow: Strelka Press, 2014.

[37] B. Sterling, Shaping Things. Cambridge, MA: MIT Press, 2005.

[38] R. van Kranenburg, The Internet of Things: A Critique of Ambient Technology and the All-Seeing Network of RFID. Amsterdam: Institute of Network Cultures, 2008. 
"I"

The contemporary precursors of networked clothing are today's wristbands, which mainly focus on fitness and health monitoring.

"I"

Networked clothes are perennially at risk of being accessed, defaced, or otherwise exploited by hackers.

\section{"1"}

CNTs can be twisted into a CNT yarn (solid-state) for the fabrication of 3-D structures through braiding, weaving, sewing, and knitting,

"초"

Recent patch antennas made from CNTs show promise at operating in the 4-9-GHz range with good gain.

\section{"I"}

An individual CNT has low scattering, high current-carrying capacity, and resistance to electromigration.
"1"

Batteries are the only current viable power source, but their weight and bulk affect wearer comfort and garment aesthetics.

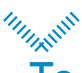

To produce high-performance stretchable piezoelectric fibers, a number of fundamental challenges must be addressed.

"

Wearable, always on, always connected fabrics quite clearly lead to clothes residing permanently in the IOT and to humans operating within a mode of constant biosurveillance.

IoT connectivity implies that clothes acquire a network address, thus becoming uniquely identifiable, and broadcast sensory data either remotely to the cloud or in an M2M mesh of devices.
Living in an environment pervasive- ly populated by networked clothes, fabrics, textiles, and other materials is fraught with the promise of a new aesthetics of the body as a fully networked object. 